\title{
Fermions without Spinors
}

\author{
I. M. Benn and R. W. Tucker \\ Department of Physics, University of Lancaster, Lancaster, England
}

\begin{abstract}
The classical Kähler equation for an inhomogeneous differential form is analysed in some detail with respect to the physical properties of its Minkowski space solutions. Although the components of the field contain only integer representations of the Lorentz group for a physical interpretation of the quantum theory, we impose fermionic commutators. The electromagnetic interactions are identical to those of a Dirac spinor field with an extra fourfold degeneracy. Possibilities for the interpretation of the extra degrees of freedom are discussed.
\end{abstract}

\section{Introduction}

This paper is concerned with a field system possessing certain remarkable properties. Some of these properties will be analysed mathematically and an attempt made to relate them to physical properties shared by fermions and bosons. The dynamics of the field system to be considered was first postulated by Kähler [1] in 1961 although there is some evidence to suggest that Darwin was aware of a similar description [2] in 1928. Apart from one notable exception [3] these ideas appear to have received little further attention. In view of the current enthusiasm for multicomponent field systems describing bosons and fermions and because of its natural formulation in arbitrary pseudo-Riemannian spaces, we feel that this system deserves further investigation.

It is notable that the classical field theories underlying all modern descriptions of the fundamental interactions between elementary particles can be formulated in terms of differential forms on space-time; i.e. tensors with antisymmetric components. This reflects the property that they can be derived from an action principle which may be globalised with the aid of differential forms. Internal degrees of freedom associated with the particles may be incorporated by using forms that take values in appropriate vector spaces, particularly Lie algebras.

When the Stern-Gerlach experiment was interpreted in terms of the intrinsic spin of the electron, this degree of freedom was also coded into an internal state 
space known as spinor space. The relativistic Dirac equation related this notion to the Lorentz group with the introduction of a 4-component spinor that carried a reducible representation of $\mathrm{SL}(2, C)$, covering $\mathrm{SO}(3,1)$, the Lorentz invariance group of the Minkowski metric describing flat space-time.

With the incorporation of gravitational interactions with other fields it became necessary to sharpen the notion of a spinor considerably since the gravitational effects according to Einstein were attributed to a non-flat space-time manifold. Such a manifold is said to carry a spinor structure [4] if the bundle of orthonormal frames with Lorentz structure group can be globally lifted into a bundle of spinor frames carrying a structure group that covers the Lorentz group. The characteristic properties of spinors under $\mathrm{SO}(3)$ rotations of $2 \pi$ can then be given an unambiguous meaning at any point of the manifold. However, the existence of a spinor structure is a topological property that is not shared by all manifolds.

The transport of spinors is defined with respect to a spinor connection naturally induced from a connection on the bundle of orthonormal frames. In a theory of interacting fields it is most natural to regard the connection and metric as among the set of independent dynamical degrees of freedom. It is well known that theories involving gravitation and spinors constructed in this way lead to geometries for space-time that have an economical description in terms of a connection with torsion, and physically imply the existence of self-interactions among the spinors. Thus the topology of the underlying manifold and the presence of a spin $(1,3)$ connection with torsion are significant features in the traditional formulation of field theories involving interactions between fermions and gravity.

In the following we enquire to what extent the notion of "intrinsic spin" can be accommodated without the notion of a spinor and the associated topological constraints that arise in arbitrary gravitational fields. Since all the finite dimensional representations of the Lorentz covering group are known, it could appear fruitless to search for fermionic characteristics by limiting attention to the tensor representations of the Lorentz group. Nonetheless by broadening the notion of a physical field to include an inhomogeneous differential form (that is, a physical field which is a sum of $p$-forms of different degree) as suggested by Kähler, one can formulate a field equation [1] which shares many of the properties in Minkowski space commonly ascribed to a spinor satisfying the Dirac equation.

In Sect. I we derive the Kähler equation from a simple action principle on an arbitrary space-time manifold. The theory involves a complex inhomogeneous differential form coupled to gravity using only the underlying space-time metric $g$ and coupled minimally to electromagnetism with the aid of the 1-form $A$. After establishing a certain amount of technology required for our further analysis we investigate in Sect. II the classical properties of the theory and point out the need for a proper quantum interpretation. The stress-energy 3-forms are derived and closed forms associated with electric current and energy-momentum conservation are discussed in Minkowski space. Using a complete basis of solutions to the equations in this space we present a straightforward quantisation in Sect. III that enables an identification of the particle states associated with the second quantised field. In order to better understand the nature of all the states described in these solutions we analyse in Sect. IV a non-relativistic reduction to a Pauli-Schrödinger 
equation in the presence of a magnetic field. In this way one can distinguish between the angular momentum and "intrinsic spin" of the system.

We conclude by pointing out that although several interpretations are possible, the equation itself possesses properties that may be of some significance when one contemplates the inclusion of gravitational interactions. Although such effects may be currently unobservable in 4-dimensions, higher dimensional versions of this equation may offer a new method of incorporating internal fermionic degrees of freedom in a natural geometric framework analogous to the Riemannian description recently given [5] for non-abelian bosonic interactions. It is pointed out that the Kähler equation also exhibits solutions that are interpretable, at least classically, in terms of purely bosonic fields. That one equation, involving only the metric properties of the underlying manifold, contains so much information in its solutions is one of the prime motivations behind our study. Indeed the system superficially resembles a superfield in which the anti-commuting basis elements are constructed solely from the basis of the exterior algebra of the cotangent space of the underlying space-time. It consequently contains a minimum of extraneous structure, couples naturally to Einsteinian gravity with a metric-compatible torsion-free connection and may not be without relevance to supersymmetric interactions.

\section{The Field Equation}

The theory to be discussed is formulated in terms of differential forms on spacetime $M$ and intrinsic operations constructed from the maps:

$$
\begin{gathered}
d: \Lambda^{p}(M) \rightarrow \Lambda^{p+1}(M), \\
*: \Lambda^{p}(M) \rightarrow \Lambda^{4-p}(M), \\
i_{X}: \Lambda^{p}(M) \rightarrow \Lambda^{p-1}(M), \quad X \in T_{x} M,
\end{gathered}
$$

where $\Lambda^{(p)}(M)$ is the space of $p$-forms on $M, d$ is the exterior, $i_{X}$ the interior derivative and $*$ the Hodge map defined with respect to the pseudo-Riemannian metric $g$ of $T_{x} M$, the tangent space at the point $x$ of $M$. We define $i_{X}$ by

$$
i_{X} \alpha=\alpha(X)
$$

and

$$
i_{X}(\alpha \wedge \beta)=i_{X} \alpha \wedge \beta-\alpha \wedge i_{X} \beta \quad \text { for } \quad \alpha, \beta \in \Lambda^{1}(M),
$$

where $\wedge$ denotes the exterior product. Conventions differ somewhat on the precise definitions of the Hodge map. If $Y$ is an arbitrary vector field on $M$ then the metric-dual 1 -form $\tilde{Y}$ is defined by

$$
\tilde{Y}(X)=g(Y, X), \quad \forall X \in T_{x} M .
$$

For any $\alpha_{j} \in \Lambda^{1}(M)$ we define $*$ to satisfy

$$
*\left(\alpha_{1} \wedge \alpha_{2} \wedge \ldots \wedge \alpha_{q}\right)=i_{\tilde{\alpha}_{q}} \ldots i_{\tilde{\alpha}_{2}} i_{\tilde{\alpha}_{1}} * 1
$$


in terms of a choice of 4 -form volume element $\in \Lambda^{4}(M), * 1$. From the definition (I.6) one derives the useful relations :

$$
\begin{gathered}
*(\Phi \wedge A)=i_{\tilde{A}} \Phi, \\
* *=(-1)^{p(4-p)} t,
\end{gathered}
$$

where $A \in \Lambda^{1}(M), \Phi \in \Lambda(M) \equiv \bigcup_{p=0}^{4} \Lambda^{p}(M)$ and $t$ is a signature for $g$, taken to be Lorentzian here $(t=-1)$. We shall call elements of $\Lambda(M), E$-forms on $M$. The real space $\Lambda_{x}(M)$ is 16-dimensional and any complex-valued $E$-form $\Phi$ can be expanded in terms of its complex homogeneous $p$-form constituents, $\phi$ :

$$
\begin{gathered}
\Phi=\sum_{p=0}^{4} \underset{(p)}{\phi}, \\
\underset{(1)}{\phi} \equiv S_{1}(\Phi)=\sum_{j=0}^{3} \phi_{j} e^{j}, \\
\underset{(2)}{\phi} \equiv S_{2}(\Phi)=\sum_{j<k}^{3} \phi_{j k} e^{j} \wedge e^{k}, \\
\underset{(3)}{\phi} \equiv S_{3}(\Phi)=\sum_{j<k<l}^{3} \phi_{j k l} e^{j} \wedge e^{k} \wedge e^{l}, \\
\underset{(4)}{\phi} \equiv S_{4}(\Phi)=\phi_{0123} e^{0} \wedge e^{1} \wedge e^{2} \wedge e^{3} .
\end{gathered}
$$

The $e^{a}$ are an arbitrary real basis for $\Lambda_{x}^{1}(M)$, and $\phi_{j k \ldots} \in C$. The homogeneous form projectors $S_{p}$ obey the rules:

$$
\begin{gathered}
1=\sum_{p=0}^{4} S_{p} \\
S_{p} S_{q}=\delta_{p q} S_{p}, \quad \text { (no sum) } \\
i_{X} S_{p}=S_{p-1} i_{X}, \quad \forall X, \\
d S_{p}=S_{p+1} d, \\
* \mathrm{~S}_{p}=\mathrm{S}_{4-p} * .
\end{gathered}
$$

Two further important maps are the main automorphism $\eta$ and the main antiautomorphism $\xi$ on the exterior algebra of $\Lambda(M)$ :

$$
\begin{aligned}
& \eta(\alpha \wedge \beta)=\eta(\alpha) \wedge \eta(\beta), \\
& \xi(\alpha \wedge \beta)=\xi(\beta) \wedge \xi(\alpha), \\
& \eta \alpha=\sum_{p} \prod_{(p)}^{\alpha}, \\
& \xi \alpha=\sum_{p} \underset{(p)}{\xi \alpha}, \\
& \eta \underset{(p)}{\alpha}=(-1)^{p} \underset{(p)}{\alpha}, \\
& \xi \underset{(p)}{\alpha}=(-1)^{\left[\frac{p}{2}\right]_{(p)}^{\alpha},}
\end{aligned}
$$


where $\alpha, \beta \in \Lambda(M), \underset{(p)}{\alpha \in \Lambda^{p}(M)}$ and $\left[\frac{p}{2}\right]$ is the integer part of $\frac{p}{2}$. Clearly $\xi$ and $\eta$ commute with themselves and with $S_{p}$.

In addition to the exterior algebra over $\Lambda(M)$ one can define the associative Clifford algebra $C\left(\Lambda^{1}(M), g\right)$ associated with $g$. If $\alpha, \beta \in \Lambda^{1}(M)$ then this Clifford product is denoted (see Note added in proof) by $\alpha_{v} \beta$ and satisfies

$$
\alpha_{v} \beta+\beta_{v} \alpha=2 g(\tilde{\alpha}, \tilde{\beta}) \text {. }
$$

We adopt the same notation $\eta$ and $\xi$ for the morphisms obeying

$$
\begin{aligned}
& \eta\left(\alpha_{v} \beta\right)=\eta(\alpha)_{v} \eta(\beta), \\
& \xi\left(\alpha_{v} \beta\right)=\xi(\beta)_{v} \xi(\alpha),
\end{aligned}
$$

and (I.19), (I.20).

There is a natural isomorphism between $C\left(\Lambda^{1}(M), g\right)$ and the exterior algebra over $\Lambda(M)$. If $\alpha \in \Lambda^{1}(M)$ then the algebras may be related by

$$
\alpha_{v}=\alpha \wedge+i_{\tilde{\alpha}}
$$

and the general case induced from $v$ associativity and linearity. Thus for $\alpha, \beta \in \Lambda^{1}(M)$,

$$
\alpha_{v} \beta=\alpha \wedge \beta+i_{\tilde{\alpha}} \beta=\alpha \wedge \beta+g(\tilde{\alpha}, \tilde{\beta})
$$

since

$$
i_{\tilde{\alpha}} \beta=\beta(\tilde{\alpha})=g(\tilde{\beta}, \tilde{\alpha}) .
$$

Thus from the vector bundle $\Lambda^{1}(M)$ and the metric $g$ one can work with an algebra on $\Lambda(M)$ involving the products $\wedge$ and $v$ satisfying (I.25). Such an algebra is distributive with respect to addition although it is not associative. It is known as the Kähler-Atiyah algebra and will be used extensively below. We shall emphasise the exterior $\wedge$ properties of the algebra when integrating differential forms over the manifold and stress the Clifford product aspects to draw attention to the "fermionic" content of the field dynamics. It is to be noted however that the fundamental entities that enter into the forthcoming description are given in terms of the space-time structure: its cotangent bundle and Lorentz metric. It may be regarded as a purely pseudo-Riemannian formulation.

The space-time action for any field theory is to be expressed as the integral of a 4-form over the manifold. We consider the action density 4-form

$$
\underset{(4)}{\Lambda}=\operatorname{Re} S_{4}\left\{\eta \Phi^{*} \wedge * d \Phi-\frac{\mu}{2} \eta \Phi^{*} \wedge * \Phi+\eta \Phi^{*} \wedge *(i e A \wedge \Phi)\right\}
$$

where $\Phi$ is a complex-valued $E$-form, $\Phi^{*}$ its complex conjugate and $A$ a real 1 -form to be associated with the electromagnetic 2 -form $F=d A$. Here $S_{4}$ is the 4-form projector defined in (I.9). This action is locally $\mathrm{U}(1)$ invariant with

$$
\begin{gathered}
\Phi \rightarrow e^{i \lambda e} \Phi, \\
A \rightarrow A-d \lambda,
\end{gathered}
$$


for $\lambda \in \Lambda^{0}(M)$. The Hodge $*$ implies a natural metric coupling to an arbitrary gravitational field. If we introduce the $\mathrm{U}(1)$ covariant derivative $D=d+i e A \wedge$ and write $S_{p}^{\prime} \equiv \operatorname{Re} S_{p}$, then (I.26) may be written :

$$
\Lambda=S_{4}^{\prime}\left\{\eta \Phi^{*} \wedge * D \Phi-\frac{\mu}{2} \eta \Phi^{*} \wedge * \Phi\right\} .
$$

We work with dimensionless $E$-forms and $\mu$ and $e$ are dimensionless real numbers. The $d$ and $*$ preserve such a dimensionless structure. One can restore all physical dimensions appropriately if equations are expressed in a local chart with dimensionless space-time coordinates $\left(\tau, x^{1}, x^{2}, x^{3}\right)$ and then transform to standard coordinates $\left(t, s^{1}, s^{2}, s^{3}\right)$ which have the physical dimensions of time and length. For this purpose one needs three standard dimensions, such as $c, \hbar$ and a reference mass $m_{0}$. The restoration of physical units follows from the transformations:

$$
\begin{gathered}
\tau=\frac{m_{0} c^{2}}{\hbar} t, \\
x^{k}=\frac{m_{0} c}{\hbar} s^{k} .
\end{gathered}
$$

If $\Lambda$ is regarded as a function of the $E$-form components $\Phi_{(N)}$, where $(N)$ is a multiindex, then a vector field $W=\sum_{(N)}\left(\delta \Phi_{(N)}\right) \frac{\partial}{\partial \Phi_{(N)}}$ on the space with local coordinates $\Phi_{(N)}$ can be used to generate arbitrary diffeomorphisms. The field equation for $\Phi$ is obtained by demanding that

$$
\mathscr{L}_{W} \Lambda=0, \quad \bmod d,
$$

for all such $W$, where $\mathscr{L}_{W}$ denotes the Lie derivative with respect to the vector field $W$. Since $\mathscr{L}_{W}$ commutes with $d$, *, and $\eta$

$$
\begin{aligned}
\mathscr{L}_{W} \Lambda= & S_{4}^{\prime}\left\{\eta \mathscr{L}_{W} \Phi^{*} \wedge * d \Phi+\eta \Phi^{*} \wedge * d \mathscr{L}_{W} \Phi-\frac{\mu}{2} \eta \mathscr{L}_{W} \Phi^{*} \wedge * \Phi-\frac{\mu}{2} \eta \Phi^{*} \wedge * \mathscr{L}_{W} \Phi\right. \\
& \left.+\eta \mathscr{L}_{W} \Phi^{*} \wedge *(i e A \wedge \Phi)+i \eta \Phi^{*} \wedge *\left(e A \wedge \mathscr{L}_{W} \Phi\right)\right\} .
\end{aligned}
$$

Since $S_{4}(\alpha \wedge * \beta)=S_{4}(\beta \wedge * \alpha)$ for any pair of $E$-forms, the second term may be written $S_{4}^{\prime}\left(d \mathscr{L}_{W} \Phi \wedge * \eta \Phi^{*}\right)$ and dropping an exact form

$$
\begin{aligned}
S_{4}^{\prime}\left(d \mathscr{L}_{W} \Phi \wedge * \eta \Phi^{*}\right) & =S_{4}^{\prime}\left(-\eta \mathscr{L}_{W} \Phi \wedge d * \eta \Phi^{*}\right), \quad \bmod d \\
& =-S_{4}^{\prime}\left(\eta \mathscr{L}_{W} \Phi^{*} \wedge d * \eta \Phi\right)
\end{aligned}
$$

Similarly the fourth term in (I.33) is $S_{4}^{\prime}\left(\frac{\mu}{2} \eta \mathscr{L}_{W} \Phi^{*} \wedge * \Phi\right)$, since $S_{4} \eta \equiv S_{4}$, and the last may be rewritten as

$$
\begin{aligned}
S_{4}^{\prime}\left(i e A \wedge \mathscr{L}_{W} \Phi \wedge * \eta \Phi^{*}\right) & =S_{4}^{\prime}\left(i e \eta \mathscr{L}_{W} \Phi \wedge A \wedge * \eta \Phi^{*}\right) \\
& =S_{4}^{\prime}\left(-i e \eta \mathscr{L}_{W} \Phi^{*} \wedge A \wedge * \eta \Phi\right)
\end{aligned}
$$


Since $W$ is arbitrary, the coefficient of $\eta \mathscr{L}_{W} \Phi^{*}$ yields the field equation

$$
* d \Phi-d * \eta \Phi-\mu * \Phi+i *(e A \wedge \Phi)-i e A \wedge * \eta \Phi=0 .
$$

Applying the map $\eta *$ to (I.36) and using $* *=-\eta$ gives

$$
-d \Phi+\delta \Phi+\mu \Phi-i e A \wedge \Phi+i *(e A \wedge * \Phi)=0,
$$

where we have introduced the co-derivative

$$
\delta \equiv * d *,
$$

which is, however, not a derivation. From (I.7) the last term in (I.37) may be reexpressed as

$$
i *(\eta * \Phi \wedge e A)=i e i_{\tilde{A}} * \eta * \Phi=-i e i_{\tilde{A}} \Phi
$$

But from (I.24)

$$
A_{v} \Phi=A \wedge \Phi+i_{\tilde{A}} \Phi
$$

thus the field equation becomes

$$
d \Phi-\delta \Phi+i e A_{v} \Phi=\mu \Phi .
$$

Introducing the further notations

$$
\begin{gathered}
\not=d-\delta, \\
D=d+i e A_{v},
\end{gathered}
$$

(I.41) can be written

$$
\not D \Phi=\mu \Phi .
$$

This is precisely the equation first studied by Kähler [1] and subsequently discussed by Graf [3].

Some simple properties of the free field system $(A=0)$ follow by noting that $d^{2}=(d-\delta)^{2}=\Delta$ is the Laplace-Beltrami operator. Hence all free field solutions to (I.44) obey

$$
\Delta \Phi=\mu^{2} \Phi .
$$

Since $\Delta$ preserves the degree of each differential form, the homogeneous components of $\Phi$ also satisfy this equation. Further, solutions of the type

$$
\Phi=\Psi+\frac{1}{\mu} d \Psi
$$

where $\Psi$ is a complex $E$-form, exist provided

$$
\delta \Psi=0 .
$$

This follows by applying $\delta$ to (I.44) and noting $\delta^{2}=0$. Consequently from (I.45)

$$
\delta d \Psi=-\mu^{2} \Psi .
$$

For example if $\Psi$ is a 0 -form one recognises that (I.48) is the scalar Klein-Gordan field equation while for $\Psi$ a 1-form (I.47), (I.48) describe a massive Proca field. 


\section{Classical Currents}

In reference [1] Kähler noted that in Minkowski space (I.44) admitted solutions in a Coulomb potential $A=\frac{e}{r} d t$ that were in exact correspondence with those found in the Dirac theory of the hydrogen atom. Subsequently Graf [3] identified Minkowski space Dirac solutions with Kählerian solutions lying in minimal left ideals of the Clifford algebra $C\left(\Lambda^{1}(M), g\right)$. The decomposition of $C\left(\Lambda^{1}(M), g\right)$ into minimal left ideals can be characterised by a set $\left\{P_{i}\right\}$ of minimal rank idempotent projectors :

$$
\begin{gathered}
\sum_{i} P_{i}=1, \\
P_{i v} P_{j}=P_{i} \delta_{i j}, \quad(\text { no sum }),
\end{gathered}
$$

and in general there exist many sets corresponding to different decompositions. It can be seen that the Kähler equation has Minkowski space solutions which lie in minimal left ideals by noting that

$$
d=e^{a} \nabla_{X_{a}}
$$

where $\nabla$ is the Riemannian connection. Furthermore these minimal left ideals are four dimensional and thus (I.44) may be decoupled into 4 sets of equations. In each set the components of the minimal ideals are coupled in a way that is isomorphic to the coupling between the four components of a spinor that satisfies the Dirac equation.

At the classical level of analysis there is no problem with restricting the class of solutions to a minimal left ideal and it appears remarkable that all the results of the Dirac equation in an electromagnetic field then follow from (I.44). However, the Dirac theory acquires proper cogency only when quantised, and one naturally expects a complete basis of solutions to be relevant for the second quantisation of (I.44). Furthermore in a space with an arbitrary gravitational field it is no longer possible to decouple the equation into sets that involve members projected into minimal ideals [3].

To prepare the ground for a physical interpretation of a complete set of solutions to (I.44) we first examine some classical currents associated with Killing symmetries of Minkowski space and the covariance of the theory under local U(1) transformations.

From the identity

$$
d S_{3}^{\prime}(\eta \Phi \wedge * \Psi+\Psi \wedge * \eta \Phi)=S_{4}^{\prime}(\eta \Phi \wedge * d \Psi-\eta d \Phi \wedge * \Psi)
$$

involving any complex $E$-forms $\Phi$ and $\psi$ it follows that if they also satisfy the Kähler equation then the generic 3-form

$$
j=S_{3}^{\prime}\left\{\eta \Phi^{*} \wedge * \Psi+\Psi \wedge * \eta \Phi^{*}\right\}
$$

is closed, that is

$$
d j=0 .
$$


The electromagnetic current 3-form, which is the coefficient of arbitrary $A$ variations of (I.26), is the closed 3 -form

$$
j_{\mathrm{e} . \mathrm{m} .}=e S_{3}^{\prime}\left\{i \eta \Phi^{*} \wedge * \Phi\right\} \text {. }
$$

If $g$ has Killing vectors $K_{i}$, and $\mathscr{L}_{K_{\imath}} F=0$, then if $\Phi$ is a solution to (I.44) so is $\mathscr{L}_{K_{i}} \Phi$. Thus there exists a closed 3 -form $j_{K_{i}}$ for each $K_{i}$

$$
j_{K_{i}}=S_{3}^{\prime}\left\{\eta \Phi^{*} \wedge * \mathscr{L}_{K_{l}} \Phi+\mathscr{L}_{K_{i}} \Phi \wedge * \eta \Phi^{*}\right\} .
$$

These 3-forms may be related to the stress 3-forms, $\tau_{a}$, defined from the action $\Lambda$ by

$$
\mathscr{L}_{Z} \Lambda=\tau_{a} \wedge \mathscr{L}_{Z} e^{a}
$$

where $Z$ generates arbitrary frame variations. For the action (I.26)

$$
\tau_{a}=S_{3}^{\prime}\left\{\Phi^{*} \wedge i_{a} * D \Phi-i_{a} D \Phi \wedge * \eta \Phi^{*}-\frac{\mu}{2} \Phi^{*} \wedge i_{a} * \Phi+\frac{\mu}{2} i_{a} \Phi \wedge * \eta \Phi^{*}\right\},
$$

where $i_{a} \equiv i_{X_{a}}$ and $\left\{X_{a}\right\}$ constitute a $g$-orthonormal frame. The $\tau_{a}$ constitute the classical sources for the coupling of the system to Einsteinian gravitation via

$$
R_{a b} \wedge *\left(e^{a} \wedge e^{b} \wedge e_{c}\right)=G \tau_{c}
$$

where the $R_{a b}$ are the curvature 2-forms. For any solution to (I.44)

$$
j_{K_{\imath}}=-2 K_{i}^{a} \tau_{a}+d S_{2}^{\prime}\left(\Phi^{*} \wedge i_{K_{i}} * \Phi+i_{K_{\imath}} \Phi \wedge * \eta \Phi^{*}\right),
$$

where $K_{i}=K_{i}^{a} X_{a}$.

For physical interpretation it is useful to perform a $3+1$ decomposition of forms on spacetime with respect to a time-like vector and an orthogonal subspace tangent to a 3-manifold $M_{3}$. In Minkowski space coordinates it is convenient to write

$$
g=-d \tau \otimes d \tau+\hat{g}
$$

in terms of the Euclidean metric $\hat{g}$ on $T_{x} M_{3}$. If $\hat{*}$ is the Hodge map defined with respect to $\hat{g}$ then the three dimensional electric current $J$ and charge density $\varrho$ are, respectively, 2 and 0 -forms on $M$ satisfying

$$
\begin{gathered}
\frac{i_{\partial}}{\partial \tau} J=0, \\
j_{\text {e.m. }}=J \wedge d \tau+\hat{*} \varrho .
\end{gathered}
$$

The total electric charge corresponding to any classical solution is

$$
Q=\int_{M_{3}} \hat{*} \varrho .
$$

We obtain an expression for $\varrho$ by performing a $3+1$ decomposition of the $E$-form $\Phi$ by writing

$$
\Phi=\Phi^{(+)}+\Phi^{(-)}
$$

with

$$
\Phi^{(\varepsilon)}=\alpha^{(\varepsilon)}+i \varepsilon d \tau \wedge \eta \alpha^{(\varepsilon)}
$$


where $\varepsilon= \pm 1$ and $\alpha^{(\varepsilon)}$ is an $E$-form on $M$ satisfying

$$
i_{\frac{\partial}{\partial \tau}} \alpha^{(\varepsilon)}=0
$$

One might refer to $\alpha^{(\varepsilon)}$ as a time dependent $E$-form on $M_{3}$. Each $\Phi^{(\varepsilon)}$ satisfies

$$
\Phi_{v}^{(\varepsilon)} P_{\varepsilon}=\Phi^{(\varepsilon)},
$$

where

$$
P_{\varepsilon}=\frac{1}{2}(1+i \varepsilon d \tau)
$$

is a right idempotent projector, since $\Phi^{(\varepsilon)}=2 \alpha^{(\varepsilon)}{ }_{v} P_{\varepsilon}$. The charge density $\varrho$ may be obtained from the definitions (II.6) and (II.14) in terms of Clifford products,

$$
\varrho=-\frac{1}{2} e S_{0}^{\prime}\left(i \bar{\Phi}_{v} d \tau_{v} \Phi\right),
$$

where it is convenient to define

$$
\bar{\Phi}=\xi \eta \Phi^{*}
$$

The bar operation on $v$ products between $E$-forms obeys

$$
\overline{\alpha_{v} \beta}=\xi \eta\left(\alpha_{v} \beta\right)^{*}=\bar{\beta}_{v} \bar{\alpha}
$$

Noting that

$$
* \Phi^{(\varepsilon)}=\hat{*} \alpha^{(\varepsilon)} \wedge d \tau+i \varepsilon \hat{*} \eta \alpha^{(\varepsilon)}
$$

use of (II.21) produces

$$
\hat{*} \varrho^{(\varepsilon)}=-\varepsilon e S_{3}^{\prime}\left(\alpha^{(\varepsilon)} * \wedge \hat{*} \alpha^{(\varepsilon)}\right)
$$

Since

$$
S_{3}^{\prime}\left(\alpha^{*} \wedge \hat{*} \alpha\right)=\sum_{p=0}^{3} S_{p}\left(\alpha^{*}\right) \wedge \hat{*} S_{p}(\alpha),
$$

and each term in the sum on the right is positive, we observe that solutions of the form $\Phi^{(\varepsilon)}$ satisfying (II.19) yield a charge density of definite sign specified by $\varepsilon$.

The closed energy-momentum 3-form is obtained by inserting the Killing vector $\frac{\partial}{\partial \tau}$ in (II.7),

$$
j_{\frac{\partial}{\partial \tau}}=S_{3}^{\prime}\left\{\eta \Phi^{*} \wedge * \mathscr{L}_{\frac{\partial}{\partial \tau}} \Phi+\mathscr{L}_{\frac{\partial}{\partial \tau}} \Phi \wedge * \eta \Phi^{*}\right\}
$$

This may be written in $3+1$ form as

$$
j \frac{\partial}{\partial \tau}=\mathbb{P} \wedge d \tau+\hat{*} \mathbb{H},
$$

where $\mathbb{P}$ is the momentum 2-form which satisfies

$$
\frac{i \partial}{\partial \tau} \mathbb{P}=0
$$


and $\mathrm{IH}$ is the Hamiltonian density 0 -form. The $\mathrm{IH}$ may also be expressed in terms of Clifford products,

$$
\mathrm{IH}=-\frac{1}{2} S_{0}^{\prime}\left(\Phi_{v} d \tau_{v} \mathscr{L}_{\frac{\partial}{\partial \tau}} \Phi\right)
$$

Inserting the $3+1$ decomposition of $\Phi$, (II.17), into (II.30) produces

$$
\hat{*} \mathbb{H}=\varepsilon S_{3}^{\prime}\left(\alpha^{(\varepsilon)^{*}} \wedge \hat{*} i \mathscr{L} \frac{\partial}{\partial \tau} \alpha^{(\varepsilon)}\right) .
$$

For a solution $\Phi^{(\varepsilon)}$ with definite frequency $\omega$ we have

$$
\mathscr{L}_{\frac{\partial}{\partial \tau}} \alpha^{(\varepsilon)}=i \omega \alpha^{(\varepsilon)}
$$

and thus for a given $\varepsilon$ it is necessary to restrict the sign of $\omega$ to keep the Hamiltonian positive definite. This feature has a counterpart in the classical Dirac theory of the electron, where the resolution is afforded by introducing creation and annihilation operators for all solutions and choosing an algebra for such operators that is consistent with an arbitrary number of quanta associated with them being described by a positive definite Hamiltonian. Using the appropriately ordered version of the current density as an operator in a Fock space then specifies the nature of the electrically charged quanta.

\section{Projectors and Quantisation}

In the preceding section it was noted that Minkowski space solutions to the Kähler equation could be decomposed into minimal left ideals, which were characterised by a set of idempotent projectors. In this section a complete set of solutions will be sought, and as a prelude we will decompose an arbitrary $E$-form into minimal left ideals. In the analysis of the energy and electric charge the projectors $P_{\varepsilon}$, defined in (II.20), proved useful. We will therefore seek projectors $P_{\sigma}$, $\sigma= \pm 1$, such that

$$
\begin{gathered}
\sum_{\sigma} P_{\sigma}=1, \\
P_{\sigma v} P_{\sigma^{\prime}}=P_{\sigma} \delta_{\sigma \sigma^{\prime}}, \quad(\text { no sum }), \\
P_{\sigma v} P_{\varepsilon}=P_{\varepsilon v} P_{\sigma},
\end{gathered}
$$

for then $P_{\sigma v} P_{\varepsilon}$ will project out minimal left ideals. A suitable choice is

$$
P_{\sigma}=\frac{1}{2}\left(1+i \sigma d x^{1} \wedge d x^{2}\right) .
$$

If $\Phi$ is an arbitrary $E$-form we may write

$$
\Phi=\sum_{\varepsilon= \pm 1} \sum_{\sigma= \pm 1} \Phi^{(\varepsilon, \sigma)},
$$

where

$$
\Phi^{(\varepsilon, \sigma)}=\Phi^{(\varepsilon, \sigma)}{ }_{v} P_{\sigma v} P_{\varepsilon} .
$$


We seek solutions to the free Kähler equation $(A=0)$ of the form

$$
\Phi^{(\varepsilon, \sigma)}=e^{i v f} \phi^{(\varepsilon, \sigma)},
$$

where $f \in \Lambda^{0}(M), v= \pm 1$ and $d \phi^{(\varepsilon, \sigma)}=\delta \phi^{(\varepsilon, \sigma)}=0$. Inserting (III.7) into (I.44) we see that we have a solution provided

$$
\phi^{(\varepsilon, \sigma)}=\frac{1}{2}\left(1+\frac{i v d f}{\mu}\right){ }_{v} \phi^{(\varepsilon, \sigma)} .
$$

If

$$
P_{v}(f)=\frac{1}{2}\left(1+\frac{i v d f}{\mu}\right)
$$

then it will satisfy the projector conditions

$$
\begin{gathered}
\sum_{v= \pm 1} P_{v}(f)=1, \\
P_{v}(f)_{v} P_{v^{\prime}}(f)=P_{v}(f) \delta_{v v^{\prime}}, \quad(\text { no sum }),
\end{gathered}
$$

if

$$
d f_{v} d f=g(\tilde{d f}, \tilde{d f})=-\mu^{2}
$$

Choosing an $f$ to satisfy this condition enables each $\phi^{(\varepsilon, \sigma)}$ to be subdivided into a pair of sets labelled by the index $v$. We seek a further subdivision by means of another left projector $P_{\lambda}(s), \lambda= \pm 1, s \in \Lambda^{0}(M)$, such that

$$
\begin{gathered}
P_{\lambda}(s)_{v} P_{v}(f)=P_{v}(f)_{v} P_{\lambda}(s), \quad \forall \lambda, v, \\
P_{\lambda}(s)_{v} P_{\lambda^{\prime}}(s)=\delta_{\lambda \lambda^{\prime}} P_{\lambda}(s), \quad \text { (no sum), } \\
\sum_{\lambda= \pm 1} P_{\lambda}(s)=1 .
\end{gathered}
$$

These conditions are met if

$$
P_{\lambda}(s)=\frac{1}{2}\left(1+\lambda d s_{v} z\right)
$$

where $z \equiv * 1$ and $d s$ is chosen to satisfy

$$
\begin{aligned}
& g(\widetilde{d s}, \widetilde{d s})=1, \\
& g(\widetilde{d f}, \widetilde{d s})=0 .
\end{aligned}
$$

Since $d f$ may be termed a momentum 1-form in the case of plane waves with $f=k_{i} x^{i}+E \tau \equiv \mathbf{k} \cdot \mathbf{x}+E \tau$, we see that $d s$ is a space-like 1 -form orthogonal to $d f$ and is expected to be related to a polarisation vector. Since $P_{\lambda}(s)_{v} P_{v}(f)$ is an idempotent left projector for each $\lambda, v$ it splits the elements $\Phi^{(\varepsilon, \sigma)}$ of each minimal left ideal into four $E$-forms labelled by $v$ and $\lambda$,

$$
\Phi^{(\varepsilon, \sigma)}=\sum_{\nu= \pm 1} \sum_{\lambda= \pm 1} \Phi_{(v, \lambda)}^{(\varepsilon, \sigma)}(s, f),
$$


where the sixteen complex $E$-forms $\Phi_{(v, \lambda)}^{(\varepsilon, \sigma)}(s, f)$ satisfy the condition

$$
\Phi_{(v, \lambda)}^{(\varepsilon, \sigma)}(s, f)=P_{v}(f)_{v} P_{\lambda}(s)_{v} \Phi_{(v, \lambda)}^{(\varepsilon, \sigma)}(s, f)_{v} P_{\varepsilon v} P_{\sigma}
$$

We may now Fourier expand an arbitrary solution of the Kähler equation into plane waves,

$$
\Phi=\sum_{\mathbf{k}} \sum_{\varepsilon} \sum_{\sigma} \sum_{v} \sum_{\lambda} \phi_{(v, \lambda)}^{(\varepsilon, \sigma)}(s, \mathbf{k}) b_{(v, \lambda)}^{(\varepsilon, \sigma)}(\mathbf{k}) e^{i v f}
$$

with $f=\mathbf{k} \cdot \mathbf{x}+E \tau, E=\left|\sqrt{\left(\mathbf{k}^{2}+\mu^{2}\right)}\right|$, and $s$ is understood to satisfy (III.15) and (III.16). The expansion (III.19) may be inserted into (II.30) to compute the total Hamiltonian. Noting that $\bar{P}_{\varepsilon}=P_{\varepsilon}, \bar{P}_{\sigma}=P_{\sigma}$ and that $S_{0}\left(\alpha_{v} \beta\right)=S_{0}\left(\beta_{v} \alpha\right)$, we may use the orthogonality properties of $P_{\varepsilon v} P_{\sigma}$ to obtain

$$
\begin{aligned}
H= & \int_{M_{3}} \hat{*} \mathbb{H} \\
= & -\frac{1}{2} \int \hat{*} 1 S_{0}^{\prime}\left\{\sum_{\varepsilon, \sigma} \sum_{v, \lambda} \sum_{v^{\prime}, \lambda^{\prime}} \sum_{\mathbf{k}, \mathbf{k}^{\prime}} i v^{\prime} E\left(\mathbf{k}^{\prime}\right) b_{(v, \lambda)}^{(\varepsilon, \sigma)^{\dagger}}(\mathbf{k}) b_{\left(v^{\prime}, \lambda^{\prime}\right)}^{(\varepsilon, \sigma)}\left(\mathbf{k}^{\prime}\right) e^{i\left(v^{\prime} f^{\prime}-v f\right)}\right. \\
& \left.\cdot \bar{\phi}_{(v, \lambda)}^{(\varepsilon, \sigma)}(\mathbf{k})_{v} d \tau_{v} \phi_{\left(v^{\prime}, \lambda^{\prime}\right)}^{(\varepsilon, \sigma)}\left(\mathbf{k}^{\prime}\right)\right\}
\end{aligned}
$$

Since

$$
\int_{M_{3}} \hat{*} 1 e^{i\left(v^{\prime} f^{\prime}-v f\right)}=e^{i\left(v^{\prime}-v\right) E(\mathbf{k})}(2 \pi)^{3 / 2} \delta\left(\mathbf{k}^{\prime}-v v^{\prime} \mathbf{k}\right),
$$

and $E\left(v v^{\prime} \mathbf{k}\right)=E(\mathbf{k})$, then

$$
\begin{aligned}
H= & -\frac{(2 \pi)^{3 / 2}}{2} S_{0}^{\prime}\left\{\sum_{\varepsilon, \sigma} \sum_{v, \lambda} \sum_{v^{\prime}, \lambda^{\prime}} \sum_{\mathbf{k}} i v^{\prime} e^{i\left(v^{\prime}-v\right) E(\mathbf{k}) \tau} E(\mathbf{k}) b_{(v, \lambda)}^{(\varepsilon, \sigma)^{\dagger}}(\mathbf{k}) b_{\left(v^{\prime}, \lambda^{\prime}\right)}^{(\varepsilon, \sigma)}\left(v v^{\prime} \mathbf{k}\right)\right. \\
& \left.\cdot \bar{\phi}_{(v, \lambda)}^{(\varepsilon, \sigma)}(\mathbf{k})_{v} d \tau_{v} \phi_{\left(v^{\prime}, \lambda^{\prime}\right)}^{(\varepsilon, \sigma)}\left(v v^{\prime} \mathbf{k}\right)\right\} .
\end{aligned}
$$

If we write $\phi_{(v, \lambda)}^{(\varepsilon, \sigma)}$ as $\phi_{(v, \lambda)}^{(\varepsilon, \sigma)}=P_{v v} P_{\lambda v} \phi_{(v, \lambda)}^{(\varepsilon, \sigma)}$ then we may use

$$
\bar{P}_{v}(\mathbf{k})_{v} d \tau_{v} P_{v^{\prime}}\left(v v^{\prime} \mathbf{k}\right)=-\frac{i v}{\mu} E(\mathbf{k}) \delta_{v v^{\prime}} P_{v}(\mathbf{k}), \quad(\text { no sum }),
$$

to arrive at

$$
H=-\frac{(2 \pi)^{3 / 2}}{2} \sum_{\varepsilon, \sigma} \sum_{\nu, \lambda} \sum_{\mathbf{k}} \frac{E(\mathbf{k})^{2}}{\mu} b_{(v, \lambda)}^{(\varepsilon, \sigma)}(\mathbf{k})^{\dagger} b_{(v, \lambda)}^{(\varepsilon, \sigma)}(\mathbf{k}) S_{0}^{\prime}\left(\overline{\phi_{(v, \lambda)}^{(\varepsilon, \sigma)}}(\mathbf{k})_{v} \phi_{(v, \lambda)}^{(\varepsilon, \sigma)}(\mathbf{k})\right) .
$$

We may express the total charge, $Q$, in a similar way

$$
\begin{aligned}
Q & =\int_{M_{3}} \hat{*} \varrho \\
& =-\frac{e}{2}(2 \pi)^{3 / 2} \sum_{\varepsilon, \sigma} \sum_{v, \lambda} \sum_{\mathbf{k}} \frac{v E(\mathbf{k})}{\mu} b_{(v, \lambda)}^{(\varepsilon, \sigma)^{\dagger}}(\mathbf{k}) b_{(v, \lambda)}^{(\varepsilon, \sigma)}(\mathbf{k}) S_{O}^{\prime}\left\{\phi_{(v, \lambda)}^{(\varepsilon, \sigma)}(\mathbf{k})_{v} \overline{\phi_{(v, \lambda)}^{(\varepsilon, \sigma)}}(\mathbf{k})\right\} .
\end{aligned}
$$

It remains to choose a normalisation by fixing $S_{0}^{\prime}\left\{\overline{\phi_{(v, \lambda)}^{(\varepsilon, \sigma)}}(\mathbf{k})_{v} \phi_{(v, \lambda)}^{(\varepsilon, \sigma)}\right\}$, although the signs of these numbers are not at our disposal. However, reference to (II.25) shows 
that the sign of $Q$ is determined by $\varepsilon$, and hence we may normalise such that

$$
S_{0}^{\prime}\left\{\phi_{(v, \lambda)}^{(\varepsilon, \sigma)}(\mathbf{k})_{v} \phi_{(v, \lambda)}^{(\varepsilon, \sigma)}(\mathbf{k})\right\}=\frac{2 \varepsilon v \mu}{(2 \pi)^{3 / 2} E(\mathbf{k})}
$$

Thus finally

$$
\begin{gathered}
H=-\sum_{\varepsilon, \sigma} \sum_{v, \lambda} \sum_{\mathbf{k}} \varepsilon v E(\mathbf{k}) b_{(v, \lambda)}^{\dagger(\varepsilon, \sigma)}(\mathbf{k}) b_{(v, \lambda)}^{(\varepsilon, \sigma)}(\mathbf{k}), \\
Q=-e \sum_{\varepsilon, \sigma} \sum_{v, \lambda} \sum_{\mathbf{k}} \varepsilon b_{(v, \lambda)}^{\dagger}\left(\begin{array}{c}
(\varepsilon, \sigma) \\
(v)
\end{array}\right) b_{(v, \lambda)}^{(\varepsilon, \sigma)}(\mathbf{k}) .
\end{gathered}
$$

In passing to the quantum theory the Fourier amplitudes $b_{(v, \lambda)}^{(\varepsilon, \sigma)}(\mathbf{k})$ become operators on some Fock space of states. At this stage we seek commutation relations for these operators that will enable us to interpret (III.26) and (III.27) in terms of number operators, so that acting on a Fock space state the Hamiltonian has positive definite eigenvalues. This may be achieved if we set

$$
\begin{aligned}
& b_{(+1, \lambda)}^{(+1, \sigma)} \equiv b_{\lambda}^{\sigma^{\dagger}}, \\
& b_{(-1, \lambda)}^{(+1, \sigma)} \equiv B_{\lambda}^{\sigma}, \\
& b_{(-1, \lambda)}^{(-1, \sigma)} \equiv d_{\lambda}^{\sigma^{\dagger}}, \\
& b_{(+1, \lambda)}^{(-1, \sigma)} \equiv D_{\lambda}^{\sigma},
\end{aligned}
$$

satisfying

$$
\left[\square_{\lambda}^{\sigma}(\mathbf{k}), \square_{\lambda^{\prime}}^{\dagger \sigma^{\prime}}\left(\mathbf{k}^{\prime}\right)\right]_{+}=\delta_{\mathbf{k}, \mathbf{k}^{\prime}} \delta^{\sigma, \sigma^{\prime}} \delta_{\lambda, \lambda^{\prime}}
$$

for

$$
\square_{\lambda}^{\sigma} \equiv\left\{b_{\lambda}^{\sigma}, d_{\lambda}^{\sigma}, B_{\lambda}^{\sigma}, D_{\lambda}^{\sigma}\right\},
$$

with all other anti-commutators zero. The ground state $|0\rangle$ is defined by

$$
b_{\lambda}^{\sigma}(\mathbf{k})|0\rangle=D_{\lambda}^{\sigma}(\mathbf{k})|0\rangle=B_{\lambda}^{\sigma}(\mathbf{k})|0\rangle=D_{\lambda}^{\sigma}(\mathbf{k})|0\rangle=0 .
$$

In terms of the number operator $N\left(\square_{\lambda}^{\sigma}\right)=\square_{\lambda}^{\sigma^{\dagger}} \square_{\lambda}^{\sigma}$ the quantum representations of the operators $H$ and $Q$ in the second quantised version of the theory are then

$$
\begin{gathered}
H=\sum_{\sigma, \lambda} \sum_{\mathbf{k}} E(\mathbf{k})\left\{N\left[b_{\lambda}^{\sigma}(\mathbf{k})\right]+N\left[D_{\lambda}^{\sigma}(\mathbf{k})\right]+N\left[B_{\lambda}^{\sigma}(\mathbf{k})\right]+N\left[d_{\lambda^{\prime}}^{\sigma}(\mathbf{k})\right]\right\}, \\
Q=e \sum_{\sigma, \lambda} \sum_{\mathbf{k}}\left\{N\left[b_{\lambda}^{\sigma}(\mathbf{k})\right]+N\left[D_{\lambda}^{\sigma}(\mathbf{k})\right]-N\left[d_{\lambda}^{\sigma}(\mathbf{k})\right]-N\left[B_{\lambda}^{\sigma}(k)\right]\right\} .
\end{gathered}
$$

Of the sixteen field quanta half of them can be distinguished by their electric charge. For $v=+1$, states labelled by $\varepsilon= \pm 1$ have positive charge while $v=-1$ states labelled by $\varepsilon= \pm 1$ have negative charge and may be termed anti-particles. We shall argue below that we can attribute to each quantum operator in the set $\square_{\lambda}^{\sigma}$ a "left spin" with eigenvalues labelled by $\lambda= \pm 1$ and a "right spin" labelled by $\sigma= \pm 1$. The choice of quantisation rules (III.33) declares the field quanta to be fermions. In the next section we examine more closely the angular momentum carried by field solutions and the way in which "intrinsic spin" of the system manifests itself. 


\section{Angular Momentum and the Non-Relativistic Limit}

The classical angular momentum currents are obtained from (II.7) where the Killing vectors generate rotations. They may be written in $3+1$ form as

$$
j_{K_{i}}=\mathbb{K}_{i} \wedge d \tau+\hat{*} \mathbb{L}_{i},
$$

where the $K_{i}$ are the Killing vectors generating the rotation group. We may find the eigenvalues of angular momentum by means of the relation

$$
\mathscr{L}_{K} \Phi=\nabla_{K} \Phi+\frac{1}{4}\left\{d \tilde{K}_{v} \Phi-\Phi_{v} d \tilde{K}\right\},
$$

where $K$ is a Killing vector, $\tilde{K}$ its metric dual and $\nabla$ the torsion free metric compatible connection. In Minkowski space we have

$$
K_{i}=x^{j} \frac{\partial}{\partial x^{k}}-x^{k} \frac{\partial}{\partial x^{j}}, \quad i, j, k=1,2,3 \text { cyclic },
$$

and we write the associated Killing forms

$$
\sigma_{i}=d \tilde{K}_{i}=2 d x^{j} \wedge d x^{k},
$$

since the Clifford algebra of the $\sigma_{i}$ is isomorphic to the familiar algebra of the Pauli matrices. For rest states $(\mathbf{k}=0)$ we may use (IV.2) in (II.7) to obtain the angular momentum density

$$
\mathbb{L}_{i}=-\frac{1}{8} S_{0}^{\prime}\left\{\bar{\Phi}_{v} d \tau_{v}\left[\sigma_{i}, \Phi\right]_{v}\right\}
$$

where

$$
\left[\sigma_{i}, \Phi\right]_{v}=\sigma_{i v} \Phi-\Phi_{v} \sigma_{i}
$$

We may compute the angular momentum density in the $x^{3}$ direction for each of the $\Phi_{(\lambda, v)}^{(\varepsilon, \sigma)}$. Using (III.18), where we have chosen $s=x^{3}$, gives

$$
\left(d x^{1} \wedge d x^{2}\right)_{v} \Phi_{(v, \lambda)}^{(\varepsilon, \sigma)}=i \lambda v \Phi_{(v, \lambda)}^{(\varepsilon, \sigma)}
$$

and

$$
\Phi_{(v, \lambda) v}^{(\varepsilon, \sigma)}\left(d x^{1} \wedge d x^{2}\right)=-i \sigma \Phi_{(v, \lambda)}^{(\varepsilon, \sigma)}
$$

Thus

$$
\mathbb{L}_{3}=-\frac{1}{2}(\lambda v+\sigma) S_{0}^{\prime}\left\{\frac{i}{2} \Phi_{(v, \lambda) v}^{(\varepsilon, \sigma)} d \tau_{v} \Phi_{(v, \lambda)}^{(\varepsilon, \sigma)}\right\} .
$$

Consequently there are three eigenvalues $(1,-1,0)$, the last being doubly degenerate. (The normalisation of the eigenvalues is obtained by comparing with the "orbital" angular momentum.) That rest states have integer eigenvalues for angular momentum is of course entirely consistent with the Lorentz group representation content of the $E$-form $\Phi$. Furthermore it should be apparent why for each $v$ we refer to $\lambda$ as labelling a left spin and $\sigma$ a right spin. How then is the intrinsic spin appropriate to half integer representations of the Lorentz group masquerading in this theory?

The existence of the closed 3-forms (II.7) followed directly from the existence of Killing vectors. However, if $\Phi$ is a solution to the Kähler equation then so is $\Phi_{v} \sigma_{i}$, 
where the $\sigma_{i}$ were defined in (IV.4). To see this note that

$$
\begin{aligned}
\not{d}\left(\Phi_{v} \sigma_{i}\right) & =e^{a}{ }_{v} \nabla_{X_{a}}\left(\Phi_{v} \sigma_{i}\right) \\
& =\not d \Phi_{v} \sigma_{i} \quad \text { since } \nabla_{X_{a}} \sigma_{i}=0 .
\end{aligned}
$$

Thus $\Phi_{v} \sigma_{i}$ is a solution to the Kähler equation as a consequence of the interactions occurring by left Clifford multiplication. So as well as the 3-forms in (II.7), the 3-forms

$$
j_{\sigma_{i}}=S_{3}^{\prime}\left\{\eta \Phi^{*} \wedge *\left(\Phi_{v} \sigma_{i}\right)+\left(\Phi_{v} \sigma_{i}\right) \wedge * \eta \Phi^{*}\right\}
$$

are also closed. These conserved currents may be recognised as the "right spin" contribution to the total angular momentum, and so we may define

$$
j_{s}=S_{3}^{\prime}\left\{\eta \Phi^{*} \wedge\left(\mathscr{L}_{K_{\imath}} \Phi+\frac{1}{4} \Phi_{v} \sigma_{i}\right)+\left(\mathscr{L}_{K_{i}} \Phi+\frac{1}{4} \Phi_{v} \sigma_{i}\right) \wedge * \eta \Phi^{*}\right\}
$$

which is a conserved current and, for rest states, has half-integral eigenvalues.

We recall that historically half integer intrinsic spin arose from an attempt to explain the non-relativistic Stern-Gerlach experiment. A doubling of states predicted by the Schrödinger equation was invoked and a magnetic interaction based on the classical coupling of a magnetic moment to an external magnetic field devised to reproduce the experimentally observed behaviour. The Schrödinger wave-function for the free electron ceased to be a scalar under rotations and was transformed as a two-component $\mathrm{SU}(2)$ spinor in order to maintain the covariance of the Pauli-Schrödinger equation under rotations. Thus the transformation properties arose as a dynamical consequence of observing how the degeneracy of states could be resolved by an electromagnetic interaction. The existence of the new spin degrees of freedom subsequently had a profound effect on the interpretation of the periodic table. The intrinsic spin interpretation was confirmed theoretically by the relativistic Dirac equation which predicted the non-relativistic gyromagnetic ratio of the electron.

We have already pointed out that, in Minkowski space, the Kähler equation can be decoupled into 4 identical Dirac equations corresponding to a decomposition of the $E$-form $\Phi$ into a set of four minimal left ideals. (This is analogous to decoupling the Pauli-Schrödinger equation into two identical Schrödinger equations in the absence of a magnetic field.) Either one restricts physical states to one ideal, which is hard to defend in general on quantum mechanical grounds, or one must expect a four-fold degeneracy in every state predicted by the Dirac theory. Certainly this is confirmed in our analysis of the field quanta above.

To gain a clearer picture of this degeneracy it is worth investigating a nonrelativistic limit of (I.44) to a set of Pauli-Schrödinger equations in the presence of a static magnetic field.

Using the decomposition (II.17) for the $E$-form $\Phi$ we may write the Kähler equation in $3+1$ form as

$$
i \varepsilon \eta \dot{\alpha}^{(\varepsilon)}=\mathbf{d} \alpha^{(\varepsilon)}+\eta \boldsymbol{\delta} \alpha^{(\varepsilon)}-\mu \alpha^{(\varepsilon)}-i e \mathbf{A}_{v} \alpha^{(\varepsilon)}-e \varepsilon V \eta \alpha^{(\varepsilon)},
$$

where $\dot{\alpha}^{(\varepsilon)}=\frac{\partial}{\partial \tau} \alpha^{(\varepsilon)}, \boldsymbol{\delta}=\hat{*} \mathbf{d} \hat{*}$ and $\mathbf{d}$ acts in $\Lambda\left(M_{3}\right)$.

We have written the electromagnetic connection 1-form as

$$
A=\mathbf{A}+V d \tau \text {. }
$$


We adjust our datum for measuring energy levels by introducing

$$
\beta^{(\varepsilon)}=e^{i \mu \tau} \alpha^{(\varepsilon)} .
$$

The $E$-form $\beta^{(\varepsilon)}$ can be unambiguously decomposed into forms of even and odd degree,

$$
\beta^{(\varepsilon)}=\beta_{+}^{(\varepsilon)}+\beta_{-}^{(\varepsilon)}
$$

Inserting this into (IV.13) gives, for $\varepsilon=+1$,

$$
\begin{gathered}
\mathbf{d} \beta_{-}^{(+)}+\boldsymbol{\delta} \beta_{-}^{(+)}-\left(i \dot{\beta}_{+}^{(+)}+\mu \beta_{+}^{(+)}\right)=\mu \beta_{+}^{(+)}+i e \mathbf{A}_{v} \beta_{-}^{(+)}+e V \beta_{+}^{(+)}, \\
\mathbf{d} \beta_{+}^{(+)}-\boldsymbol{\delta} \beta_{+}^{(+)}+i \dot{\beta}_{-}^{(+)}=i e \mathbf{A}_{v} \beta_{-}^{(+)}-e V \beta_{-}^{(+)} .
\end{gathered}
$$

Similarly for $\varepsilon=-1$ we obtain

$$
\begin{gathered}
\mathbf{d} \beta_{-}^{(-)}+\boldsymbol{\delta} \beta_{-}^{(-)}+i \dot{\beta}_{+}^{(-)}=i e \mathbf{A}_{v} \beta_{-}^{(-)}-e V \beta_{+}^{(-)} \\
\mathbf{d} \beta_{+}^{(-)}-\boldsymbol{\delta} \beta_{+}^{(-)}-\left(i \dot{\beta}_{-}^{(-)}+\mu \beta_{-}^{(-)}\right)=\mu \beta_{-}^{(-)}+i e \mathbf{A}_{v} \beta_{+}^{(-)}+e V \beta_{-}^{(-)} .
\end{gathered}
$$

The non-relativistic limit of the pair (IV.17) and (IV.18) is derived by dropping the terms $\dot{\beta}_{+}^{(+)}$and $e V \beta_{+}^{(+)}$in (IV.17) and inserting the result into (IV.18) to obtain the Pauli-Schrödinger equation for $\beta_{-}^{(+)}$,

$$
-i \dot{\beta}_{-}^{(+)}=\frac{1}{2 \mu}\left(\mathbf{d}-\boldsymbol{\delta}-i e \mathbf{A}_{v}\right)\left(\mathbf{d}+\boldsymbol{\delta}-i e \mathbf{A}_{v}\right) \beta_{-}^{(+)}+e V \beta_{-}^{(+)} .
$$

Treating (IV.19) and (IV.20) similarly produces

$$
-i \dot{\beta}_{+}^{(-)}=\frac{1}{2 \mu}\left(\mathbf{d}+\boldsymbol{\delta}-i e \mathbf{A}_{v}\right)\left(\mathbf{d}-\boldsymbol{\delta}-i e \mathbf{A}_{v}\right) \beta_{+}^{(-)}+e V \beta_{+}^{(-)} .
$$

We may write (IV.21) and (IV.22) together as

$$
-i \dot{\beta}_{-\varepsilon}^{(\varepsilon)}=\frac{1}{2 \mu}\left(\mathbf{d}-i e \mathbf{A}_{v}\right)\left(\mathbf{d}-i e \mathbf{A}_{v}\right) \beta_{-\varepsilon}^{(\varepsilon)}+e V \beta_{-\varepsilon}^{(\varepsilon)},
$$

where $\boldsymbol{d}=d+\eta \boldsymbol{\delta}$. Dropping labels on the $\beta$ 's for clarity but remembering that they satisfy $\varepsilon \eta \beta=-\beta$, we may expand the Clifford products in (IV.23),

$$
-i \dot{\beta}=\frac{1}{2 \mu}\left\{\mathbf{d}^{2} \beta-i e \mathbf{d}\left(\mathbf{A}_{v} \beta\right)-e^{2} \mathbf{A}_{v} \mathbf{A}_{v} \beta-i e \mathbf{A}_{v} \mathbf{d} \beta\right\}+e V \beta
$$

Now

$$
\begin{aligned}
\mathbf{d}^{2} & =\mathbf{d} \eta \boldsymbol{\delta}+\eta \boldsymbol{\delta} \mathbf{d} \\
& =-(\mathbf{d} \boldsymbol{\delta}-\boldsymbol{\delta} \mathbf{d}) \eta \\
& \equiv \nabla^{2}
\end{aligned}
$$

which is the Laplace-Beltrami operator on $M_{3}$. Furthermore in terms of the Riemannian connection $\boldsymbol{\nabla}$ on $M_{3}$,

$$
\mathbf{d}\left(\mathbf{A}_{v} \beta\right)=\mathbf{d} \mathbf{A}_{v} \beta-\mathbf{A}_{v} \mathbf{d} \beta+2 \nabla_{\tilde{\mathbf{A}}} \beta,
$$


so Eq. (IV.23) can be written finally in the form

$$
-i \dot{\beta}=\frac{1}{2 \mu}\left\{\nabla^{2} \beta-i e \mathbf{A} \mathbf{A}_{v} \beta-2 i e \nabla_{\tilde{\mathbf{A}}} \beta-e^{2} \mathbf{A}_{v} \mathbf{A}_{v} \beta\right\}+e V \beta .
$$

One readily recognises in the non-relativistic Hamiltonian the magnetic-spin interaction. Indeed $\mathbf{d A}=\mathbf{d} \mathbf{A}+\eta \boldsymbol{\delta} \mathbf{A}=\mathbf{B}$, where $\mathbf{B}$ is a magnetic field 2-form on $M_{3}$ in the gauge $\boldsymbol{\delta} \mathbf{A}=0$, so the magnetic interaction Hamiltonian is

$$
H_{I}=\frac{i e}{2 \mu} \mathbf{B}_{v} .
$$

The simplest way to confirm the eigenvalues of this operator (which acts on the non-relativistic $E$-form $\beta$ via a Clifford product) is to adapt the projector $P_{\lambda}$ to the direction of the external magnetic field. Recall that $\beta$ was derived from an $\alpha$ defined by

$$
\Phi=\alpha+i \varepsilon d \tau \wedge \eta \alpha
$$

Let us write $d s=s_{0} d \tau+\hat{s}$ in terms of a 0 -form $s_{0}$ and 1 -form $\hat{s}$ with $i_{\frac{i}{\hat{i} \tau}} \hat{s}=0$. Then imposing

$$
P_{\lambda}(s)_{v} \Phi=\Phi
$$

gives

$$
\lambda\left(s_{0} * 1_{v} \alpha-i \varepsilon \hat{*} \hat{s}_{v} \eta \alpha\right)=\alpha .
$$

For rest frame solutions $s_{0}=0$ and we may choose

$$
\hat{*} \hat{s}=\frac{\mathbf{B}}{|\mathbf{B}|},
$$

so that (IV.31) becomes

$$
-i \lambda \varepsilon \frac{\mathbf{B}}{|\mathbf{B}|} v \eta \alpha=\alpha
$$

and hence

$$
-i \lambda \varepsilon \frac{\mathbf{B}}{|\mathbf{B}|} v \eta \beta=\beta \text {. }
$$

The non-relativistic $E$-forms satisfy $\varepsilon \eta \beta=-\beta$ and so (IV.34) gives

$$
i \mathbf{B}_{v} \beta=\lambda|\mathbf{B}| \beta .
$$

Thus the left projector

$$
P_{\lambda}=\frac{1}{2}\left(1+\frac{\lambda \mathbf{B}}{|\mathbf{B}|} v d \tau\right)
$$

projects out rest-frame energy eigenstates. The energy levels labelled by $\lambda= \pm 1$ are 
separated by $\frac{e}{\mu}|\mathbf{B}|$ precisely as observed in the Stern-Gerlach experiment. This justifies our nomenclature of referring to $\lambda$ as a left-spin eigenvalue or magnetic quantum number.

We have selected $v=1$ by choosing the positive frequency solution to make the non-relativistic reduction. Thus in this limit $v= \pm 1$ distinguishes particles and anti-particles and $\lambda= \pm 1$ labels conventional "half integer intrinsic spin" electromagnetic interactions. However, for each state labelled by $(v, \lambda)$ there is a 4 -fold degeneracy corresponding to the states with $\varepsilon= \pm 1, \sigma= \pm 1$. Thus unlike ordinary non-relativistic rest electrons which are conventionally considered to carry 2 -spin degrees of freedom, rest particles described in this theory appear to carry 8 degrees of freedom. A static magnetic field resolves the $\lambda= \pm 1$ degeneracy with an interaction indistinguishable from that observed experimentally for electrons if $e$ and $\mu$ are chosen appropriately. In Minkowski space the extra $(\varepsilon, \sigma)$ degeneracy is not affected by electromagnetism, since the Hamiltonian is block diagonal for any $A$. Whatever particles are to be described by (I.44) they appear to simulate charged fermions in their electromagnetic couplings and each electromagnetic state is associated with four degrees of freedom $(\varepsilon, \sigma)$ that we term right-spin.

\section{The Interpretation of Right-Spin}

A satisfactory interpretation of the Kähler equation requires a resolution of the problem of right-spin. If Eq. (I.44) is to describe, say, electrons then how are we to understand the fourfold degeneracy labelled by different values of $\varepsilon, \sigma$ ? If we restrict the interactions to electromagnetism in Minkowski space then these states are indistinguishable. We may then establish correspondence with the Dirac theory by setting

$$
b_{\lambda}^{+1 \dagger}|0\rangle=b_{\lambda}^{-1 \dagger}|0\rangle=D_{\lambda}^{+1 \dagger}|0\rangle=D_{\lambda}^{-1 \dagger}|0\rangle .
$$

However, in the presence of an arbitrary gravitational field the Kähler equation does not admit solutions lying in one of the minimal left ideals, and we would expect the fourfold degeneracy to be broken in any weak field limit, rendering (V.1) unacceptable.

It is possible to modify the Kähler equation in such a way as to give a different physical interpretation to the different ideals. Conceivably this could make a virtue out of necessity and lead to a unified description of different basic fermions. For example, we could modify the equation as follows:

$$
\text { el } \Phi=(\mu+i e A)_{v} \Phi_{v} \frac{1}{2}(1+i * 1) .
$$

If

$$
Q_{\varepsilon} \equiv \frac{1}{2}(1+i \varepsilon * 1)
$$

then

$$
Q_{\varepsilon v} Q_{\varepsilon^{\prime}}=Q_{\varepsilon} \delta_{\varepsilon \varepsilon^{\prime}}, \quad(\text { no sum }) \quad \varepsilon, \varepsilon^{\prime}= \pm 1
$$


and so we may decompose $\Phi$ uniquely as

$$
\Phi=\sum_{\varepsilon= \pm 1} \Phi^{\varepsilon}
$$

where

$$
\Phi^{\varepsilon} Q_{\varepsilon}=\Phi^{\varepsilon}
$$

Thus we may write (V.2) as

$$
\begin{gathered}
d \Phi^{+}=(\mu+i e A)_{v} \Phi^{+}, \\
d \Phi^{-}=0 .
\end{gathered}
$$

(Incidentally, the equations will decouple in this way in an arbitrary gravitational field.) Now $\Phi^{+}$is a massive electrically charged field whereas $\Phi$ is massless and neutral. The $Q_{\varepsilon}$ project $\Phi$ into non-minimal left ideas and the Kähler equation would have to be further modified if we wish to have different physical properties for all four minimal ideals. This approach will be investigated in the future.

\section{Appendix}

In this appendix an explicit basis is chosen for the construction of a complete set containing 16 plane-wave solutions for the free Kähler equation. We first select a particular basis to span the real 8-dimensional space $\Lambda_{x}\left(M_{3}\right)$. This can be constructed in terms of the idempotent projectors:

$$
\begin{gathered}
P_{\varepsilon}=\frac{1}{2}(i+i \varepsilon d t), \\
P_{\sigma}=\frac{1}{2}\left(1+i \sigma e^{12}\right), \\
\sigma= \pm 1, \quad \varepsilon= \pm 1,
\end{gathered}
$$

where we write simply $e^{12}$ for $e^{1} \wedge e^{2}=e^{1}{ }_{v} e^{2}$ for an orthonormal basis $\left\{e^{1}=d x^{1}\right.$, $\left.e^{2}=d x^{2}, e^{3}=d x^{3}\right\}$ and note $P_{\sigma v} P_{\varepsilon}=P_{\varepsilon v} P_{\sigma}$. A convenient complex basis for $A_{x}\left(M_{3}\right)$ is then $\alpha_{l}^{\sigma}(l=1,2,3,4)$, where

$$
\begin{aligned}
& \alpha_{1}^{\sigma}=e^{1}{ }_{v} P_{\sigma}, \\
& \alpha_{2}^{\sigma}=e^{31}{ }_{v} P_{\sigma}, \\
& \alpha_{3}^{\sigma}=e^{3}{ }_{v} P_{\sigma}, \\
& \alpha_{4}^{\sigma}=P_{\sigma},
\end{aligned}
$$

so an arbitrary $E$-form on $M$ can be expanded

$$
\Phi(x)=\sum_{\varepsilon} \sum_{\sigma} \sum_{l=1}^{4} A_{l}^{\varepsilon, \sigma}(x) \alpha_{l v}^{\sigma} P_{\varepsilon}
$$

in terms of 16 complex amplitudes $A_{l}^{\varepsilon, \sigma} \in C$. The basis above has the useful orthonormality property:

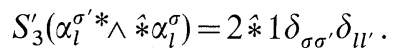


It is not difficult to find a complete set of Minkowski space plane wave solutions to (I.44) if we put to zero the electromagnetic interaction. With the ansatz

$$
\Phi^{(\varepsilon)}=\tilde{\alpha}_{v}^{(\varepsilon)} P_{\varepsilon} e^{i \omega \tau-i \mathbf{k} \cdot \mathbf{x}}
$$

in terms of $\mathbf{k}=\left(k_{1}, k_{2}, k_{3}\right)$ the Kähler equation is satisfied if

$$
i\left(\sum_{j=1}^{3} k_{j} e^{j}\right)_{v} \tilde{\alpha}^{(\varepsilon)}=(\mu-\varepsilon \omega \eta) \tilde{\alpha}^{(\varepsilon)},
$$

provided $\omega^{2}=\mathbf{k}^{2}+\mu^{2}$. In terms of the basis $\alpha_{l}^{\sigma}$, (A.10) is readily solved. A particular complete set of basis solutions may be expressed in terms of:

$$
\begin{aligned}
& \Gamma_{+}^{\{\varrho\}}(\mathbf{k})=N_{+}^{\{\varrho\}}\left(\alpha_{1}^{\sigma}+\frac{i k_{3} \alpha_{2}^{\sigma}}{\mu_{-}}+\frac{i k_{+}^{\sigma}}{\mu_{-}} \alpha_{4}^{\sigma}\right), \\
& \Gamma_{-}^{\{\varrho\}}(\mathbf{k})=N_{-}^{\{\varrho\}}\left[\alpha_{3}^{\sigma}-\frac{i k_{-} \alpha_{2}^{\sigma}}{\mu_{-}}+\frac{i k_{3}}{\mu_{-}} \alpha_{4}^{\sigma}\right],
\end{aligned}
$$

where $\{\varrho\}$ is the set of labels $\left\{\varepsilon, \sigma, \frac{\omega}{|\omega|}\right\}, N_{ \pm}^{\{\varrho\}}$ are arbitrary normalisations and

$$
\begin{gathered}
k_{ \pm}^{\sigma}=k_{1} \pm i \sigma k_{2}, \\
\mu_{ \pm}=\mu \pm \varepsilon \omega, \\
\omega= \pm E= \pm \mid \sqrt{\mathbf{k}^{2}+\mu^{2}} .
\end{gathered}
$$

It may be noted that the subscript $m= \pm 1$ on the $E$-forms $\Gamma_{ \pm}^{\{\varrho\}}$ refers to the even and odd nature of the degree of that form. Thus an arbitrary solution to (I.44) with $A=0$ may be Fourier analysed in Minkowski space as:

$$
\Phi(x)=\sum_{\mathbf{k}} \sum_{\varepsilon= \pm 1} \sum_{\sigma= \pm 1} \sum_{\frac{\omega}{|\omega|}= \pm 1} \sum_{m= \pm 1} b_{m}^{\{\varrho\}}(\mathbf{k}) I \Gamma_{m}^{\{\ell\}}(\mathbf{k}) e^{i(\mathbf{k} \cdot \mathbf{x}+\omega \tau)},
$$

where

$$
I \Gamma_{m}^{\{\varrho\}}(\mathbf{k})=(1+\varepsilon d \tau \wedge \eta)_{v} \Gamma_{m}^{\{\varrho\}}(\mathbf{k})
$$

constitute a particular basis of 16 complex eigen- $E$-forms. They satisfy the orthonormality relations:

$$
\begin{gathered}
S_{3}^{\prime}\left\{\Gamma_{m}^{(\varepsilon, \sigma, \pm)}(\mathbf{k})^{*} \wedge \hat{*} \Gamma_{m^{\prime}}^{\left(\varepsilon^{\prime}, \sigma^{\prime}, \mp\right)}(\mathbf{k})\right\}=2 N_{m}^{\{\varrho\}} N_{m^{\prime}}^{\left\{\varrho^{\prime}\right\}} \hat{*} 1 \delta_{m m^{\prime}} \delta_{\sigma \sigma^{\prime}} \delta_{\varepsilon \varepsilon^{\prime}}\left(\frac{2 E}{E \pm \mu \varepsilon}\right) \\
S_{3}^{\prime}\left\{\Gamma_{m}^{(\varepsilon, \sigma, \mp)}(\mathbf{k})^{*} \wedge \hat{*} \Gamma_{m^{\prime}}^{\left(\varepsilon^{\prime}, \sigma^{\prime}, \mp\right)}(\mathbf{k})\right\}=2 N_{m}^{\{\varrho\}} N_{m^{\prime}}^{\left\{\varrho^{\prime}\right\}} \hat{*} 1 \delta_{m m^{\prime}} \delta_{\sigma \sigma^{\prime}} \delta_{\varepsilon,-\varepsilon^{\prime}}\left(\frac{2 E}{E \pm \varepsilon \mu}\right),
\end{gathered}
$$

and hence the following normalisation suggests itself:

$$
N_{m}^{\{\varepsilon, \sigma, \pm\}}=\sqrt{\frac{E \pm \mu \varepsilon}{E}} \frac{1}{4(2 \pi)^{3 / 2}} .
$$


In the continuous limit $\sum_{\mathbf{k}}$ is replaced by an integral and the $k$ space normalisation completed as usual with

$$
\frac{1}{(2 \pi)^{3 / 2}} \int_{M_{3}} e^{ \pm i \mathbf{k} \cdot \mathbf{x}} \hat{*} 1=\delta(\mathbf{k})
$$

in terms of the Dirac distribution on $\mathbf{k}$-space.

\section{References}

1. Kähler, E.: Der innere Differentialkalkül. Rend. Mat. (3-4) 21, 425 (1962)

2. Darwin, C.G.: The wave equations of the electron. Proc. R. Soc. 118, 654 (1928)

3. Graf, W.: Differential forms as spinors. Ann. Inst. Henri Poincaré XXIX, 85 (1978)

4. See for example, Hitchin, N.: Harmonic spinors. Adv. Math. 14, 1 (1974)

5. Benn, I.M., Tucker, R.W.: Gauge field interactions from extra dimensional gravity. Phys. Lett. 112B, 344 (1982)

Benn, I.M., Tucker, R.W.: A Riemannian description of non-abelian gauge interactions. Lancaster preprint (1982)

Communicated by S. W. Hawking

Received August 15, 1982

Note added in proof: The authors regret the use of the symbol " "." to denote the Clifford product (usually denoted by $\vee$ ) and hope that the meaning is clear from the context. 